\title{
Role of magnetic resonance imaging in tumor staging and follow-up for bladder cancer
}

\author{
Ki Choon Sim^, Deuk Jae Sung^ \\ Department of Radiology, Korea University Anam Hospital, Korea University College of Medicine, Seoul, Korea \\ Contributions: (I) Conception and design: All authors; (II) Administrative support: None; (III) Provision of study materials or patients: All authors; \\ (IV) Collection and assembly of data: None; (V) Data analysis and interpretation: None; (VI) Manuscript writing: All authors; (VII) Final approval of \\ manuscript: All authors. \\ Correspondence to: Prof. Deuk Jae Sung, MD, PhD. Department of Radiology, Korea University Anam Hospital, Korea University College of \\ Medicine, 73, Goryeodae-ro, Seongbuk-gu, Seoul 02841, Korea. Email: urorad@korea.ac.kr.
}

\begin{abstract}
Urothelial carcinoma of the bladder is a common urologic malignancy. Complex factors, such as local stage, tumor grade, biologic potential, and various conditions, can affect the treatment strategy for bladder cancer. However, the local stage — in particular, the presence or absence of muscle invasionsignificantly influences decisions regarding treatment strategy. The role of cystoscopy for screening, diagnosis, and transurethral resection cannot be overlooked. The importance of local staging with magnetic resonance imaging is increasing; magnetic resonance imaging of the bladder is considered a useful staging modality. Moreover, a radiologic reporting system for evaluating and scoring muscle invasion of bladder cancer was recently released. This system is based on multiparametric magnetic resonance imaging and is also expected to be feasible for post-treatment follow-up of bladder cancer. In this review, we discuss the role of magnetic resonance imaging in the local staging of urothelial carcinoma of the urinary bladder and post-treatment imaging. In addition, several technical aspects for obtaining appropriate quality magnetic resonance images of the bladder will be discussed.
\end{abstract}

Keywords: Urothelial carcinoma; urinary bladder neoplasms; magnetic resonance imaging; stage; TNM

Submitted Oct 30, 2019. Accepted for publication May 25, 2020.

doi: 10.21037/tau-19-671

View this article at: http://dx.doi.org/10.21037/tau-19-671

\section{Introduction}

Bladder cancer is the tenth most common cancer worldwide, occurring 3.4 times more commonly in males than in females (1). Bladder cancer has a high local recurrence rate; thus, long-term surveillance is required after initial treatment. Almost all bladder neoplasms are malignant tumors, of which $95 \%$ are of epithelial origin (2). These tumors gradually invade the inside to the outside of the bladder wall. The treatment strategy for bladder cancer is mainly determined on the basis of distinction of non-muscle invasive (stage $\mathrm{T} 1$ or less) from muscle-invasive (stage $\mathrm{T} 2$ or more) tumors. For non-muscle invasive bladder cancer (NMIBC) or non-bulky tumors, transurethral resection of bladder tumor (TURBT) and intravesical therapy are commonly used $(3,4)$. In contrast, muscle-invasive bladder cancer (MIBC) is treated with radical cystectomy, radiation therapy, chemotherapy, or a combination thereof (5). Imaging evaluation is an important step in determining the treatment strategy. Computed tomography (CT) is the most commonly used and convenient imaging modality for the detection and staging of bladder cancer. It is also more costeffective than magnetic resonance imaging (MRI), and small polypoid mass can be easily diagnosed as an early stage

^ ORCID: Ki Choon Sim, 0000-0002-3344-8018; Deuk Jae Sung, 0000-0002-5025-3052. 


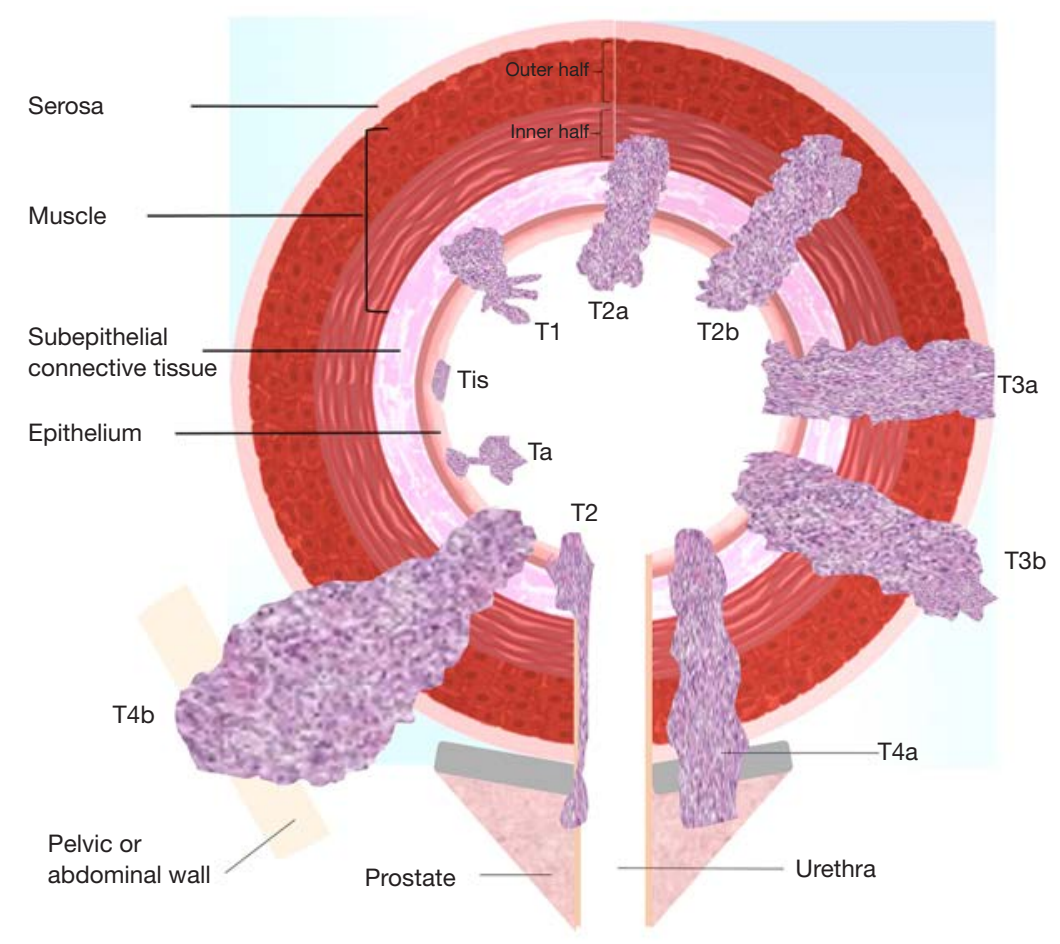

Figure 1 Diagram showing bladder wall anatomy and local tumor staging of urinary bladder cancer based on invasion depth (see Tables 1,2 for T stage definitions).

tumor without MRI. However, CT has several limitations for local tumor staging (6). MRI is being increasingly used for local staging of bladder cancer and considered the modality of choice for local staging. Moreover, a multiparametric MRI reporting system for local bladder cancer staging, the vesical imaging-reporting and data system (VI-RADS), was recently released (7).

With this background, here we discuss the role of MRI in the local staging of urothelial carcinoma of the urinary bladder by the presence or absence of muscle invasion. We also discuss the role of post-treatment follow-up bladder MRI and several technical aspects of obtaining appropriate high-quality magnetic resonance images of the bladder.

\section{Normal bladder wall anatomy and corresponding MRI appearance}

The urinary bladder, except where the peritoneum covers the superior surface (bladder dome), is mostly an extraperitoneal organ. It is composed of muscles, and its size and shape varies according to the amount of urine inside it and the adjacent organs. The trigone is an inverted triangular area between the two ureteral orifices and the internal urethral opening (8). Histologically, the four defined layers of the bladder wall are: (I) the urothelium (mucosa), which lines the bladder lumen; (II) the highly vascular lamina propria (submucosa, composed of subepithelial connective tissue); (III) the muscularis propria (composed of detrusor muscle); and (IV) the outermost serosa $(2,6,9)$ (Figure 1). The innermost layer, the urothelium, is thinner than the total thickness of the bladder wall, while the thickness of the second inner layer, the lamina propria, varies according to the degree of bladder distension $(6,9)$. Although there is overlap, the average thickness of the lamina propria also differs by location, being thinner at the trigone and thicker at the dome (10). The muscularis propria layer, called the detrusor muscle, consists of the inner longitudinal, middle circular, and outer longitudinal muscle layers. The outermost serosa is formed by a loose layer of connective tissue.

Imaging modalities like MRI or CT do not possess sufficient spatial resolution to differentiate among the histological bladder wall layers. The inner two layers are not observed on T2-weighted images (T2WI) or diffusion- 
Table 1 TNM classification of urinary bladder cancer based on the AJCC 7th and 8th edition $(9,22)$

\footnotetext{
Primary tumor $(T)$

TX: Primary tumor cannot be assessed

TO: No evidence of primary tumor

Ta: Noninvasive papillary carcinoma

Tis: Carcinoma in situ; "flat tumor"

T1: Tumor invades subepithelial connective tissue

T2: Tumor invades muscular propria
}

T2a: Tumor invades superficial muscularis propria (inner half)

T2b: Tumor invades deep muscularis propria (outer half)

T3: Tumor invades perivesical tissue

T3a: Microscopically

T3b: Macroscopically (extravesical mass)

T4: Tumor invades any of the following: prostatic stroma, seminal vesicles, uterus, vagina, pelvic wall, abdominal wall

T4a: Tumor invades prostatic stroma, uterus, vagina

T4b: Tumor invades pelvic wall, abdominal wall

Regional lymph nodes $(N)^{\ddagger}$

NX: Lymph nodes cannot be assessed

NO: No lymph node metastasis

N1: Single regional lymph node metastasis in the true pelvis (perivesical, hypogastric, obturator, external iliac, or presacral lymph node)

N2: Multiple regional lymph node metastasis in the true pelvis (same as N1)

N3: Lymph node metastasis to the common iliac lymph nodes

Distant metastasis (M)

MO: No distant metastasis

M1: Nonregional lymph node metastasis or distant organ metastases

M1a: Nonregional lymph node metastasis only: common iliac or upper retroperitoneal nodes

M1b: Non-lymph node distant metastases

${ }^{\ddagger}$, regional lymph nodes include both primary and secondary drainage regions. All other nodes above the aortic.

Table 2 Updates in the American Joint Committee on Cancer staging of urinary bladder cancer

\begin{tabular}{ll}
\hline Category & Details \\
\hline T1 & Attempt for subcategorization in the transurethral resection recommended \\
T2 & Staging of diverticular cancers has no T2 \\
T4 & Prostatic stromal invasion clarified that must be transmural from bladder: subepithelial stromal invasion staged as T2 (urethral) \\
N1 & Perivesical lymph node added \\
M1 & Divided into nonregional lymph node only (M1a) and non-lymph node distant metastases (M1b) \\
\hline
\end{tabular}



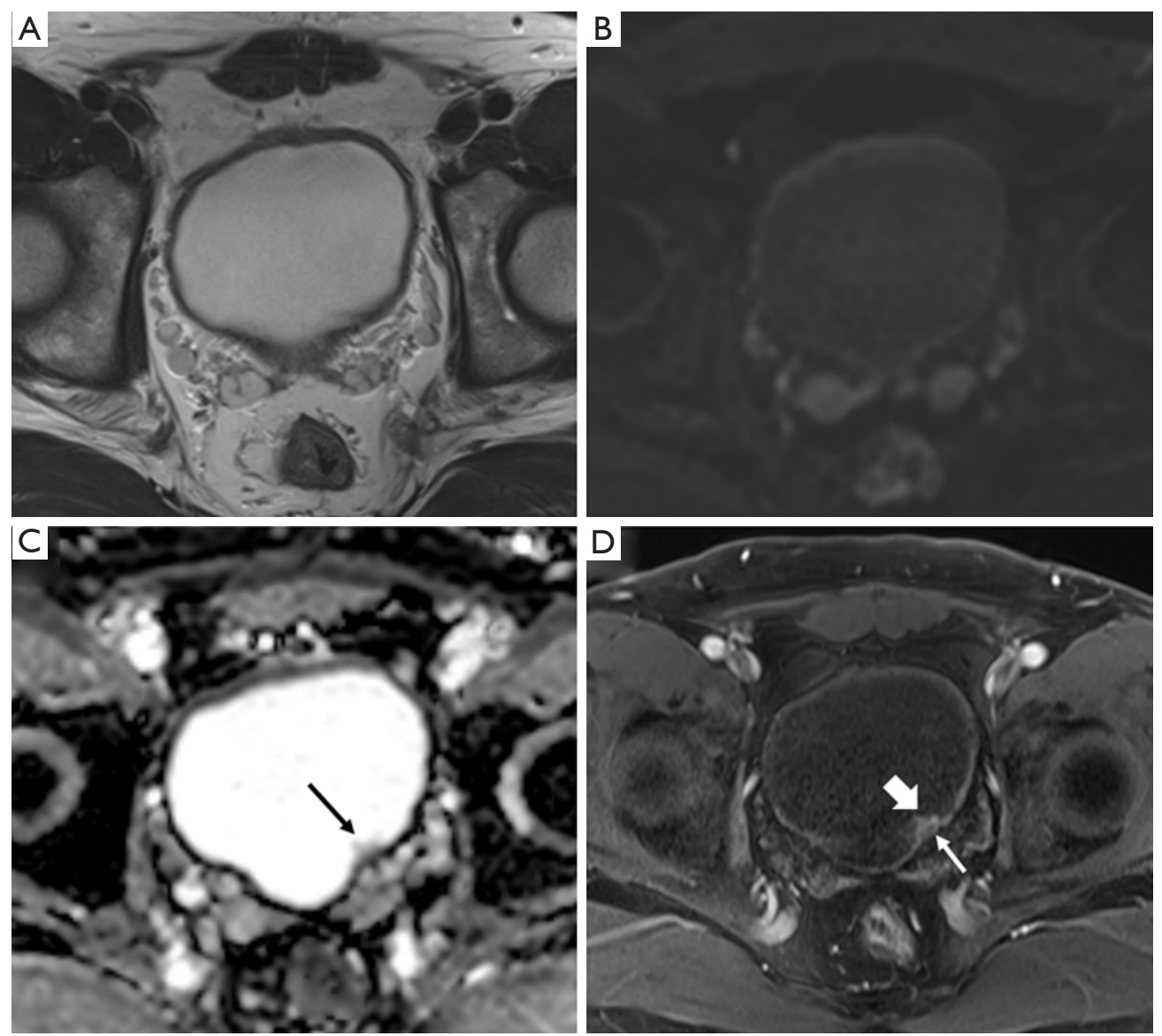

Figure 2 Urothelial carcinoma (stage Ta) in a 74-year-old man. Axial T2-weighted magnetic resonance image (T2WI) (A) showing the normal bladder wall as a hypointense line. Axial diffusion-weighted image (DWI) with a high $b$ value (B) showing the normal bladder wall as an intermediate signal intensity line. Axial apparent diffusion coefficient map (C) showing the bladder wall with low signal intensity. Small papillary lesion with intermediate signal intensity (arrow) in the left posterior wall that was not clearly visible on previous sequences. Axial three-dimensional T1-weighted spoiled gradient echo image (D) obtained 60 seconds after the administration of contrast material shows intense enhancement of the bladder tumor (thick arrow) unlike the muscle layer (thin arrow).

weighted images (DWI). The muscularis propria is observed as a hypo-signal intensity line on T2WI and as an intermediate signal intensity line on DWI. In the apparent diffusion coefficient (ADC) map, the bladder wall appears as an intermediate signal intensity line surrounding the urine, which is very bright. In dynamic contrast enhancement (DCE) sequences, the inner two layers and the muscularis propria are can possibly be distinguished; however, the muscularis propria is indistinguishable from the urothelium and lamina propria (7). In DCE sequences, the lamina propria appears abundant in vascular structures, resulting in avid early arterial enhancement, whereas the muscularis propria layer is relatively less enhanced and shows progressive delayed enhancement. The three muscle layers appear as a single layer on imaging (6). The outermost serosa is not observed on MRI; rather, perivesical fat tissue is observed (Figure 2).

\section{MRI and technical considerations for image acquisition}

MRI is considered the imaging modality of choice in the local staging of bladder cancer. Its high spatial resolution and soft-tissue contrast make it possible to distinguish the tumor from the normal detrusor muscle of the bladder wall. MRI also has better diagnostic accuracy for diagnosing perivesical tumor involvement than CT $(11,12)$. 

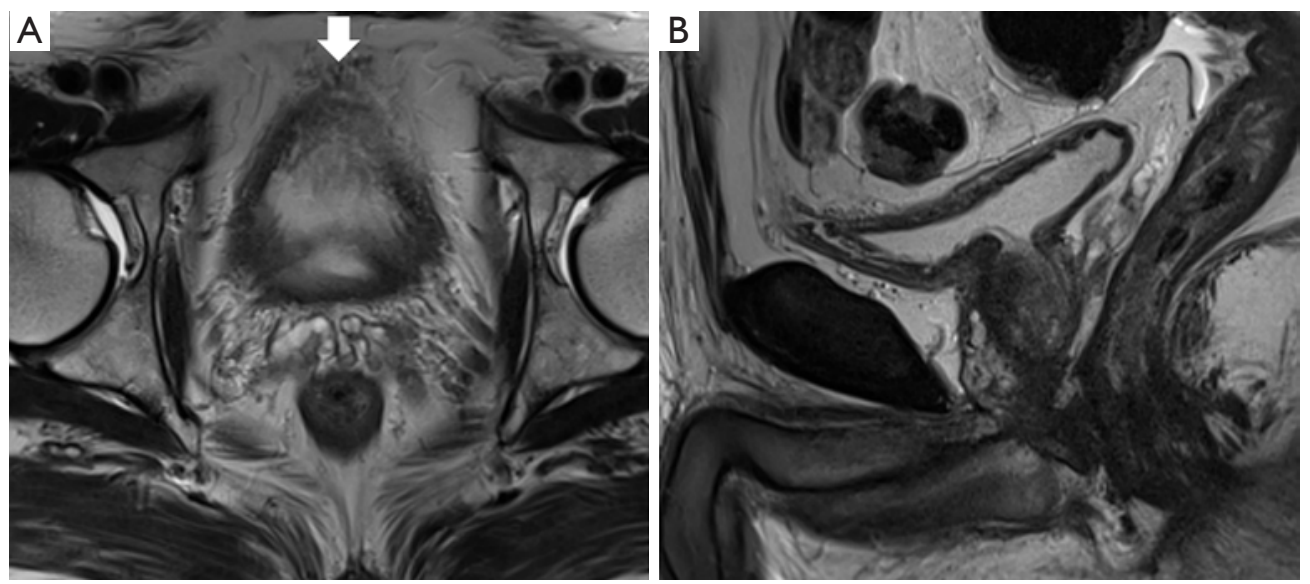

Figure 3 Magnetic resonance imaging (MRI) of a 74-year-old man who underwent transurethral resection of a bladder tumor previously. Axial (A) and sagittal high-resolution T2-weighted magnetic resonance image (B) showing a poorly distended urinary bladder. The bladder lumen is barely visible on the axial image because of insufficient distension. The vascular structures (thick arrow) between the anterior and perivesical fat mimic tumor infiltration. Evaluation of the bladder wall and lumen on the sagittal image was limited yet marginally feasible; MRI recall or additional cystoscopy should be considered in such cases.

\section{Patient preparation}

Antispasmodic agents, administered either intramuscularly or intravenously, should be used in cases without contraindications to reduce artifacts caused by bowel peristalsis. It may be convenient to administer an intravenous injection of antispasmodic agents through the line accessed for MRI contrast injection just before the MRI examination begins. Appropriate bladder distension enables discrimination of the bladder wall layers. A poorly distended bladder wall will appear thick, irregular, and uneven, leading to a misdiagnosis of bladder cancer or the over-staging of existent tumors (Figure 3). Conversely, an overdistended bladder may result in patient motion due to discomfort and can decrease sensitivity for detecting plaquelike lesions. Appropriate bladder distension is considered 300-500 mL. To ensure proper bladder filling, patients must void urine for the last time 2 hours before the MRI study or drink $500-1,000 \mathrm{~mL}$ of water 30 minutes before the study $(6,7)$. Cystoscopy and urine catheterization, which can introduce air into the bladder and cause susceptibility to artifacts during diffusion-weighted imaging, should be avoided several days before the examination.

\section{Timing of MRI}

TURBT or intravesical therapy causes edema and inflammation in the bladder wall and the surrounding perivesical tissue, making it difficult to distinguish normal tissues from bladder cancer and resulting in an overestimation of local stage $(7,8)$. Because there is no established method to avoid or measure reactive changes in the bladder wall, an MRI study is best performed before the procedure or at least 2 weeks after transurethral resection, biopsy, or intravesical treatment (7).

\section{MRI equipment}

The use of MRI (1.5 or 3.0 T) is recommended to achieve high spatial resolution and a high signal-to-noise ratio (7). However, if possible, the use of 3T MRI is recommended to achieve higher specificity in the local staging of bladder cancer (13). The use of a multichannel phased-array external surface coil is also recommended. The use of an endorectal coil can improve the spatial resolution of bladder tumors on the posteroinferior wall; however, this is not included in recent recommendations $(7,14)$.

\section{Image acquisition}

Multiparametric MRI (mpMRI) of the bladder consists of a combination of T2-, diffusion-, and T1-weighted imaging with DCE sequences. All images should be properly obtained to include various pelvic organs and pelvic lymph nodes. Depending on the institution, additional T2WI and delayed contrast-enhanced T1-weighted 

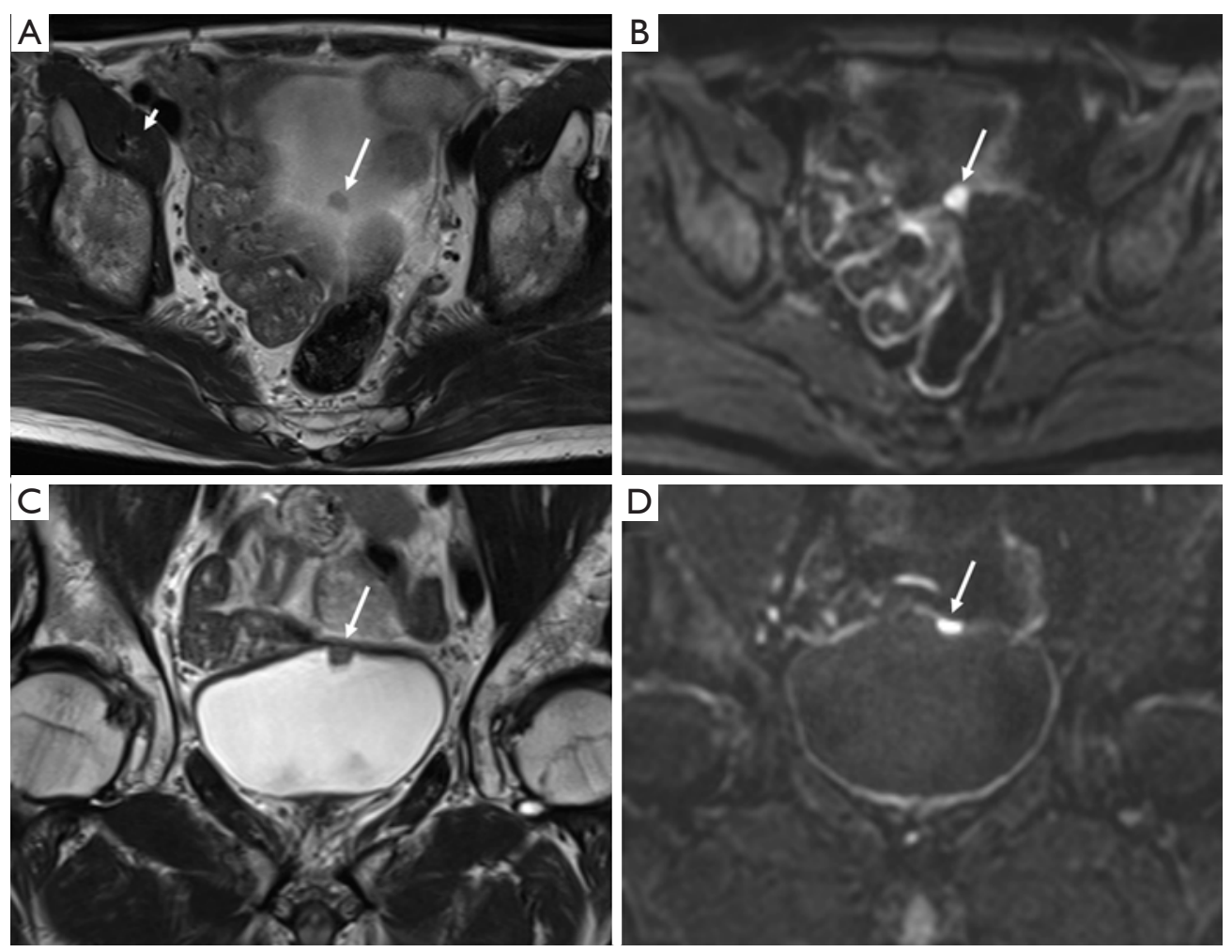

Figure 4 Urothelial carcinoma (stage T1) in a 63-year-old man. Axial T2-weighted image (T2WI) (A) showing a small tumor in the bladder dome (arrow). In the diffusion-weighted image (DWI) with a high $b$ value (B), the tumor shows markedly increased signal intensity (arrow). However, the localization of bladder tumors solely based on these images is difficult. The lesions located on the bladder dome are often not clearly visualized on axial images because of the persistence of partial volume artifacts. In such cases, coronal images (C, coronal T2WI; D, coronal DWI with high $b$ value) are extremely helpful for tumor localization and T stage evaluation.

images (T1WI) including both kidneys may be obtained to detect synchronous or metachronous urothelial tumors or hydronephrosis in the upper urinary tracts. However, for upper urinary tract evaluation, CT urography may alternatively be used $(5,8)$. It is also useful for detecting metastases to distant organs such as the liver, adrenal glands, lungs, and bones; metastasis to the peritoneal space; and upper retroperitoneal lymphadenopathy.

At least two planes of multiplanar (axial, coronal, and sagittal) T2WI without fat suppression are obtained with two-dimensional (2D) fast-spin-echo (FSE) or turbo-spinecho (TSE). If multiplanar T2WI is standard protocol, it would be better to acquire sagittal or coronal T2WI first, followed by axial T2WI to minimize possible differences in bladder distension status between the last T2WI and subsequent axial DWI (15). For 2D-FSE, a slice thickness of 3-4 $\mathrm{mm}$ is recommended; three-dimensional (3D) spinecho acquisition may be also used. Axial 2D TSE or FSE
T1WI is used to detect hemorrhagic components in the bladder and bone metastases. It is recommended that the same parameters as those of T2WI, such as slice thickness, field-of-view, and matrix, be used.

Diffusion-weighted imaging is a functional imaging technique reflecting proton diffusion properties in water molecules within the tissues. It plays an important role in pelvic MRI including mpMRI of the bladder. Usually only DWI of the axial plane is obtained, but that of the coronal or sagittal planes should also be obtained to ensure an accurate evaluation of tumors occurring in various parts of the bladder considering its spherical shape (Figures 4,5). However, it is better to obtain at least two planes such as in T2WI because it takes longer to obtain all three planes in DWI. A high $b$ value $\left(800-1,000 \mathrm{~s} / \mathrm{mm}^{2}\right)$ is essential for visualizing bladder cancer with high contrast to the surrounding tissue and urine. In addition, an image with a low $b$ value, such as $b=0$ or $50 \mathrm{~s} / \mathrm{mm}^{2}$, must also be acquired 

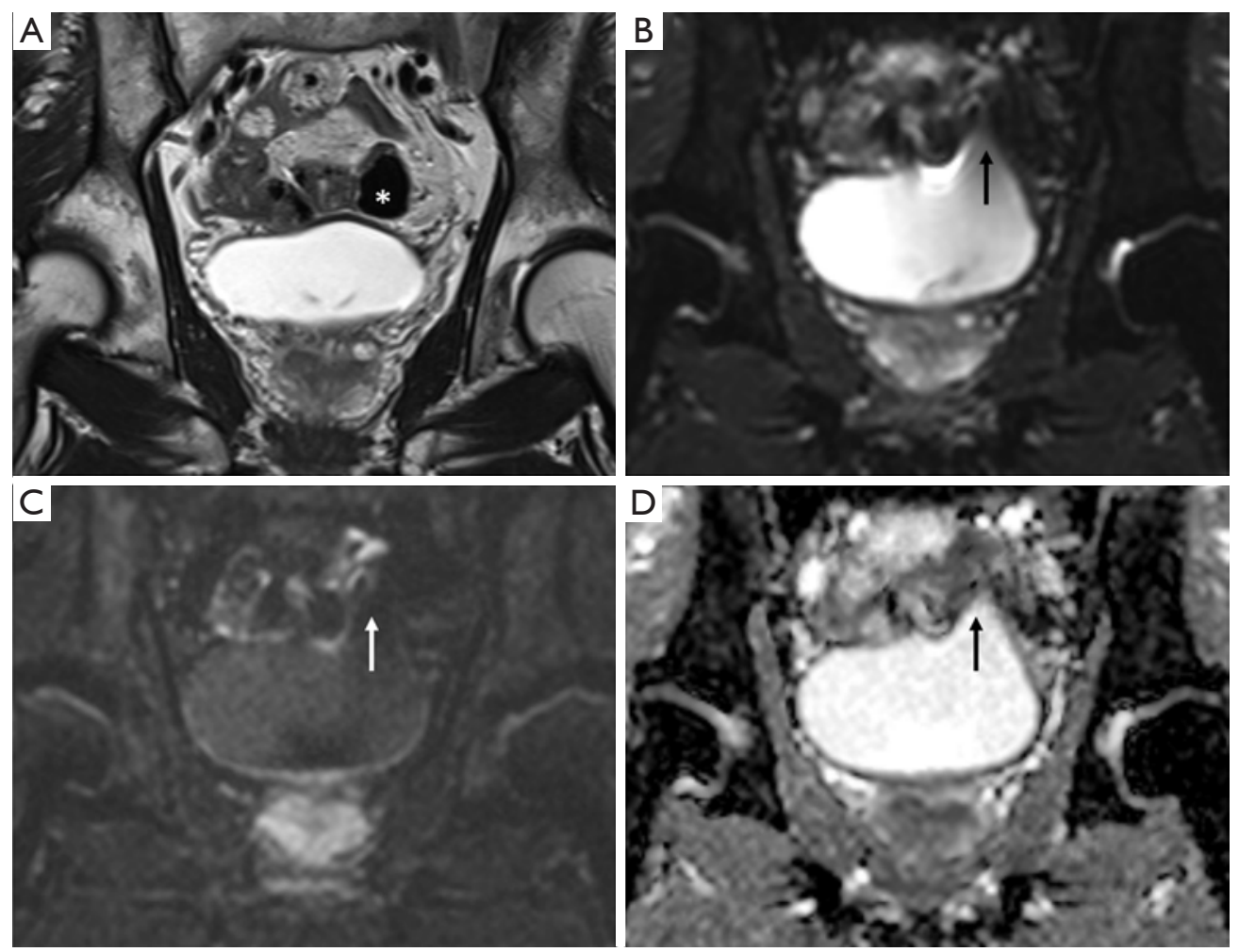

Figure 5 Vulnerability on a coronal diffusion-weighted imaging. Additional slice of the coronal plane image of the patient described in Figure 4. (A) T2-weighted image (T2WI), (B) $b=0$, (C) $b=1,000$, and (D) apparent diffusion coefficient map. Coronal T2WI showing no abnormality in the left dome portion, and an air-filled bowel structure (asterisk) with dark signal intensity is located immediately adjacent to it. However, in the diffusion-weighted images, distortion is observed in the left dome of the bladder (arrow). It is believed that motion artifacts caused by bowel peristalsis and susceptibility artifacts caused by air existed simultaneously.

to obtain the ADC map.

For DCE imaging, a 2D or 3D T1 gradient echo sequence with fat suppression may be used, but $3 \mathrm{D}$ acquisition (e.g., VIBE, LAVA, THRIVE) is preferred to obtain higher spatial resolution. In addition to the FSE or TSE T1WI mentioned earlier, obtaining additional fat-suppressed pre-contrast T1WI is recommended. A gadolinium-based contrast agent is administered using a power-injector system at a dose of $0.1 \mathrm{mmol} / \mathrm{kg}$ of body weight at a rate of $1.5-2.0 \mathrm{~mL} / \mathrm{s}$ if a standard relaxivity agent is used, followed by a saline flush (7). The first contrast enhancement image should be acquired about 30 seconds after starting the contrast injection, followed by the same sequences four to six times every 30 seconds to inspect rapid enhancement of a tumor compared with that of a normal bladder wall. The order of image acquisition is also important. If possible, the DCE sequence should be performed last, after the T2WI or DWI acquisitions. If diffusion-weighted imaging is performed after DCE, the excreted MRI contrast agent may change the urine signal (Figure 6). In addition, the iodine contrast agent used in contrast-enhanced CT before MRI increases the T1 signal of urine, which may affect image interpretation. For similar reasons, the late phase (e.g., 3 minutes or more) is not useful for $T$ staging, and the contrast between the bladder wall and the tumor is decreased (Figure 7).

\section{MRI findings of bladder cancer}

T2-weighted imaging provides optimal soft-tissue contrast among the bladder tumor, urine, and bladder wall. The muscularis propria layer appears as a hypointense band against marked hyperintense intraluminal urine and perivesical fat. A tumor usually exhibits intermediate signal intensity on T2WI. Therefore, T2-weighted imaging can be a key sequence for the evaluation of muscle invasion and 

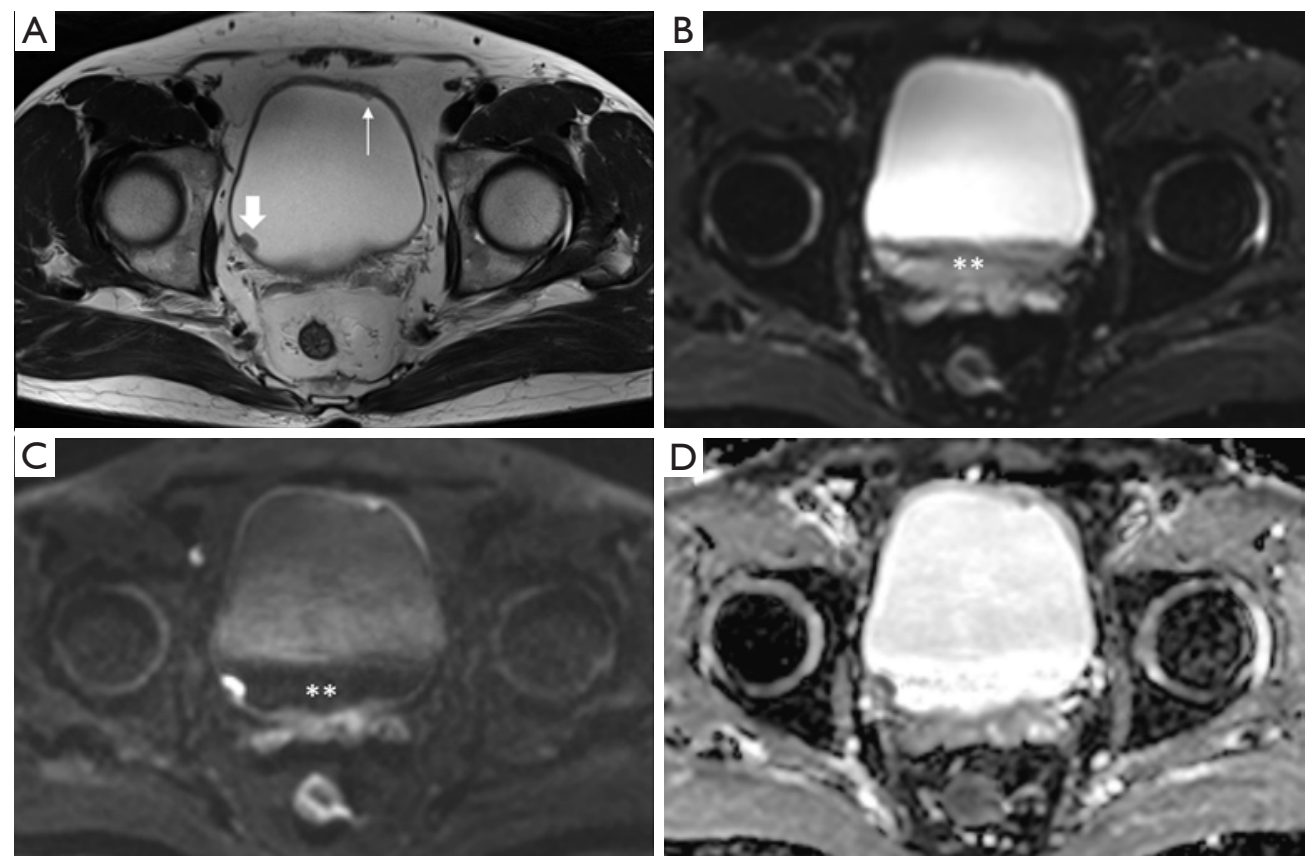

Figure 6 Urothelial carcinoma (stage T1) in a 65-year-old man highlighting the importance of magnetic resonance (MR) image acquisition order. (A) A small tumor (about $1 \mathrm{~cm}$ ) is visible in the right posterior wall (thick arrow), while another tiny polypoid lesion is seen in the left anterior wall (thin arrow) on an axial T2-weighted image. Diffusion-weighted image (B, $b=0 ; \mathrm{C}, b=800)$ and apparent diffusion coefficient map (D) obtained after delayed contrast enhancement shows a new heterogeneous low signal in the bladder-dependent portion (asterisk) that differs from the signal of urine in the nondependent portion. This represents the change in the signal intensity of urine because of excretion of the MR contrast agent that, to some extent, influences the contrast between the right posterior tumor and urine in the low $b$ value image (B).
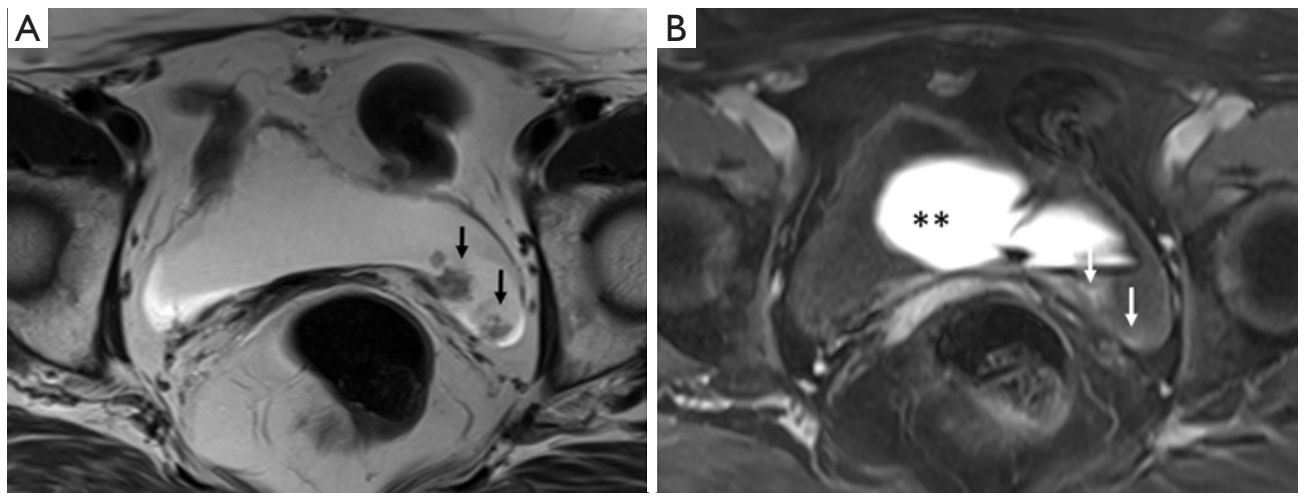

Figure 7 Urothelial carcinoma in a 68-year-old woman. Axial T2-weighted image (T2WI) (A) showing two papillary masses in the left posterior wall of bladder (arrow). Axial three-dimensional T1-weighted spoiled gradient echo image (B) obtained 300 seconds after contrast agent administration showing marked hyperintensity due to excreted magnetic resonance contrast agent in the urinary bladder (asterisk). The urine, which has been filling the bladder, has a dark signal intensity. In this case, the tumor is localized on the left posterior wall and cannot be visualized on delayed contrast-enhanced images (arrow). 
perivesical extension. MRI is considered superior to CT for differentiating NMIBC from MIBC (16). In NMIBC, the hypointense muscle layer is preserved; however, in MIBC, the hypointense muscle layer is disrupted by a T2 intermediate signal intensity tumor. Coronal or sagittal planes based on axial planes can aid accurate staging. However, even with MRI, the differentiation of T2a and T2b tumors is almost impossible. In addition, it is difficult to accurately distinguish between muscle invasion and microscopic perivesical fat invasion (T2 vs. T3a) in some cases. Since the rate of lymph node metastasis increases in T2b tumors, an accurate diagnosis is required for cystectomy specimens (17). On T2WI or T1WI without fat suppression, extravesical tumor extension appears as a nodular or hairy soft-tissue projection with intermediate signal intensity in high signal intensity perivesical fat. One study reported that the sensitivity and specificity of T2WI for diagnosing T3b tumors were up to $100 \%$ and 94\% compared with those of CT (18). However, chemical shift artifacts can occur between the bladder wall and the perivesical fat and obscure the bladder wall. This can also be misinterpreted by perivesical tumor infiltration (19).

Similar to T2WI, DWI/ADC cannot distinguish between the inner two layers, i.e., the urothelium and lamina propria, in the normal bladder. Occasionally, when there is wall thickening associated with edema rather than the tumor, the inner two layers are observed as a single layer with T2 high signal intensity (20). The muscularis propria shows intermediate signal intensity on high $b$ value DWI and high signal intensity on the corresponding ADC map and is interpreted as no diffusion restriction. However, if the tumor is hyperintense on high $b$ value DWI and hypointense on the corresponding ADC map, it is interpreted as diffusion restriction. When the bladder tumor invades the muscularis propria or perivesical fat, the tumor signal also extends to the muscularis propria or perivesical fat (Figure 8) (7). In T1 or less tumors, stalks with no diffusion restriction can be identified as masses with diffusion restriction. Alternatively, T1 or less tumors can be diagnosed even when there is thickening in the lamina propria to which the stalk is connected and there is no diffusion restriction (Figure 9) (7,21). ADC value and tumor grade are generally inversely related. For example, when there is a tumor with a similar high signal intensity in the high $b$ value image on diffusion-weighted imaging, a tumor with a lower ADC value (darker on the image) is suggested to be high grade (Figure 10). According to a recent study, an ADC cutoff of $<0.9 \times 10^{-3} \mathrm{~mm}^{2} / \mathrm{s}$ to distinguish between high- and low-grade tumors showed a sensitivity of $91.7 \%$, specificity of $60 \%$, and area under the curve of $0.74(95 \%$ CI, 0.641-0.825) (21). A lack of conclusive ADC cutoff values, differences in MRI equipment, and differences in applied techniques (different $b$ values) limit this semiquantitative tool. The study also states that MRI cannot replace tumor grading, which is defined using transurethral resection biopsy (21).

The diffusion-weighted imaging has the inherent disadvantages of a low signal-to-noise ratio and spatial resolution, which limit its usefulness for the evaluation of small tumors or a thin bladder wall with overdistension and leaves it vulnerable to various artifacts. Moreover, it is also important to differentiate tumors from benign conditions such as blood clots, infected urine, and abscesses that may show marked diffusion restriction (16). Therefore, to ensure accurate lesion interpretation, the use of a sequence such as T2-weighted imaging or DCE should also be considered.

On DCE MRI, tumors show rapid bright enhancement and can be distinguished from normal muscularis propria, which does not simultaneously show contrast enhancement. In early-stage cancer ( 11 or less), the tumor stalk is preserved and delayed contrast enhancement is observed (Figure 9D). The absence of early enhancement of the muscularis propria is suggestive of non-muscle invasive tumors. However, in tumors with muscle-invasive potential, the tumor stalk is not observed or early enhancement of the tumor is observed to extend into the low signal intensity muscularis propria. Like T2WI and DWI, it is important for assessing muscle invasiveness $(7,19)$.

\section{Local staging of urothelial carcinoma of the bladder}

Most patients with bladder cancer present with painless hematuria. The initial evaluation is expected to vary by institution, but CT urography, cystoscopy, or both tests are performed simultaneously. Each modality plays a complementary role and has its own strengths. If the tumor is small or the likelihood of a superficial tumor is very high on cystoscopy, TURBT may be performed without MRI. However, as mentioned earlier, mpMRI of the bladder plays an important role in $\mathrm{T}$ staging and is considered an essential pre-treatment test method (7).

Invasion depth through the layers of the bladder wall is the most important factor for local $\mathrm{T}$ staging of urothelial carcinoma of the bladder. The TNM system, based on the American Joint Committee on Cancer (AJCC) $7^{\text {th }}$ edition, 

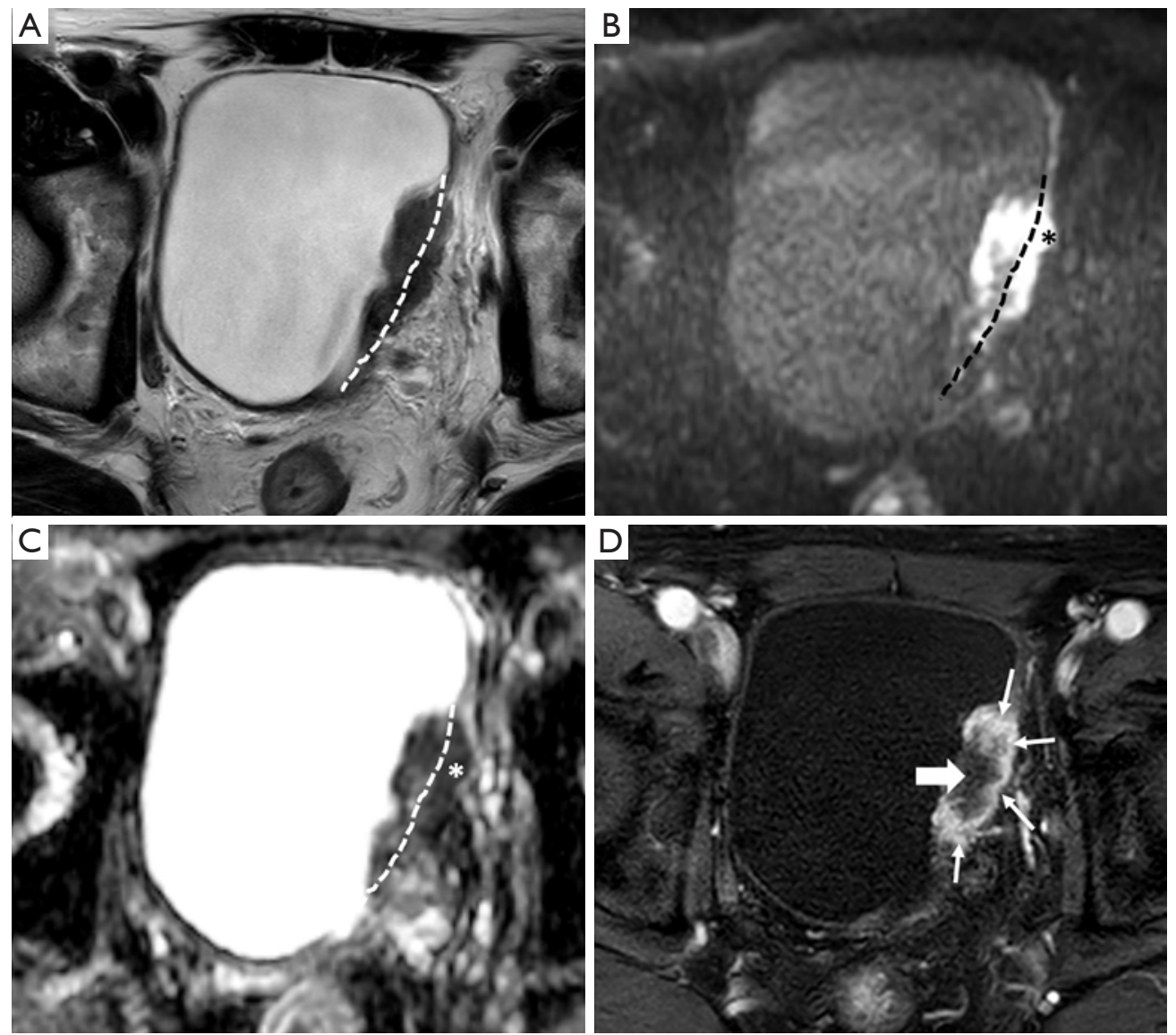

Figure 8 Urothelial carcinoma (stage T3b) in a 79-year-old man. Axial T2-weighted image (A) shows a T2 heterogeneous mass with intermediate to low signal intensity in the left wall. If the dotted line is considered the boundary between the bladder wall and the perivesical fat, a tumorous lesion extending into the perivesical fat area is observed, which can be staged as T3b. A high $b$ value image (B) and apparent diffusion coefficient maps (C) also show diffusion restriction in the tumors extending to the bladder wall (asterisk). Axial three-dimensional T1-weighted spoiled gradient echo image (D) obtained 40 seconds after contrast agent administration shows early peripheral enhancement (thin arrow) and central necrosis (thick arrow) of the large tumor. This case is classified as vesicle imaging-reporting and data system grade 5 .

is widely used in the staging of urothelial carcinoma of the bladder (Figure 1, Table 1) (22). In 2018, the AJCC $8^{\text {th }}$ edition was released with some modifications (9). In this edition, transurethral resection was recommended for the subcategorization of $\mathrm{T} 1$ cancer, and there was no $\mathrm{T} 2$ in the staging of diverticular cancer that does not contain the muscularis propria layer. In cases of prostate invasion, transmural invasion and subepithelial stroma invasion should be staged as T4a and T2, respectively (Table 2) (9).

The mpMRI of the bladder, which refers to a combination of T2-weighted imaging, diffusion-weighted imaging, and DCE sequences, is used in the scoring of the recently proposed Vesical Imaging-Reporting and Data System (VIRADS) (7). It is important to declare a five-point scoring system that can predict muscle-invasive tumors in the pretransurethral resection setting. Tumors smaller than 1 $\mathrm{cm}$ are highly unlikely to involve muscle invasion and are assigned VI-RADS 1 . Tumors larger than $1 \mathrm{~cm}$ are assigned VI-RADS 2 (Figure 11). Tumors with a high possibility of muscle invasion or perivesical tumor extension are assigned VI-RADS 4 or 5 (Figure 8). In our experience, tumors considered VI-RADS 1 , 4, or 5 were not significantly difficult to interpret on MRI. A recent study that validated the VI-RADS also reported that VI-RADS scores of 4 or 5 were $100 \%$ accurate for predicting muscle invasion (3). VIRADS 3 is assigned to cases with an equivocal likelihood of muscle invasion. Muscle invasion was reportedly present in two-thirds of patients assigned VI-RADS 3 (Figure 12) (3). 

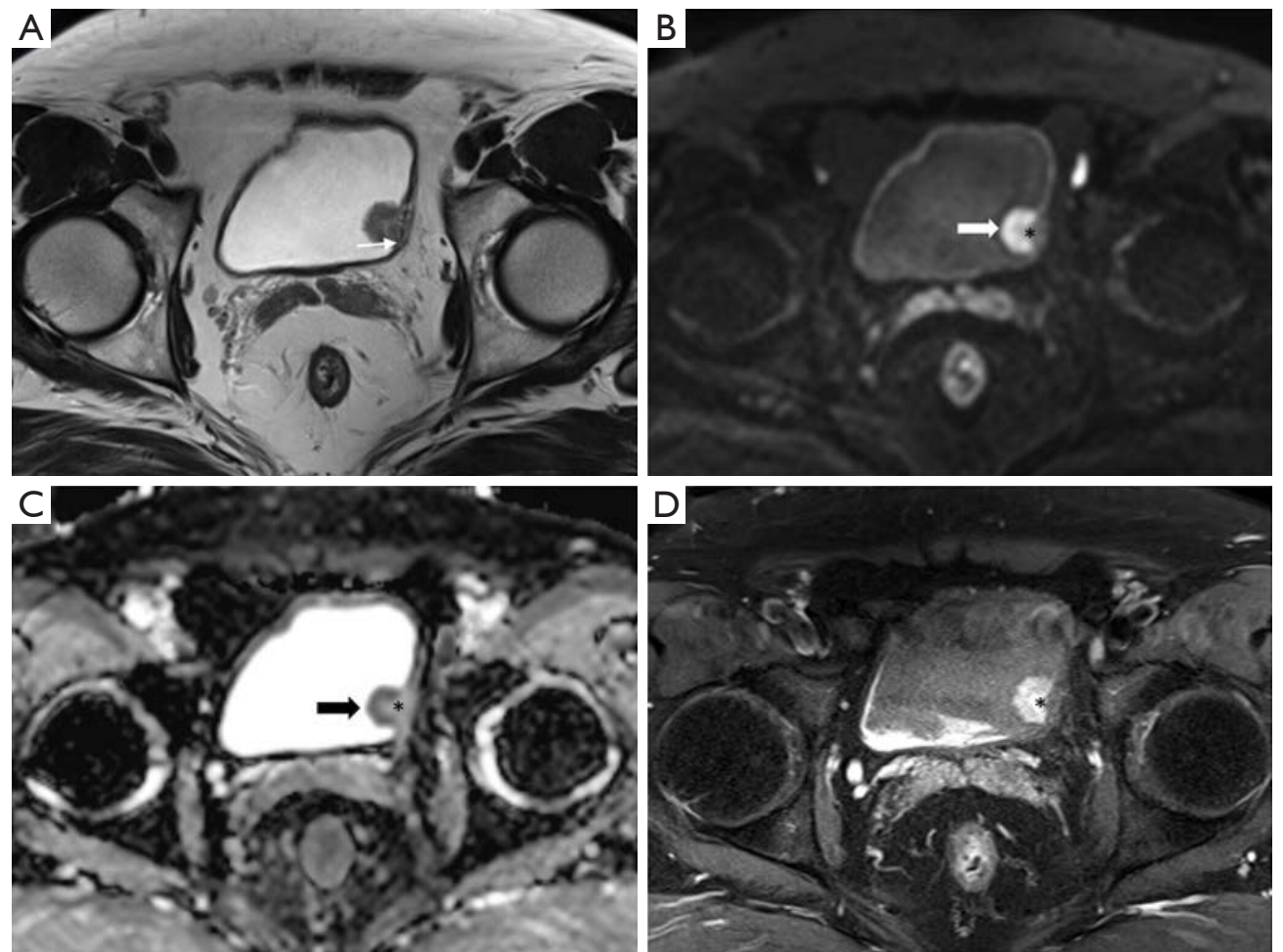

Figure 9 Urothelial carcinoma (stage T1) in a 60-year-old man. Axial (A) T2-weighted images showing a tumor in the left bladder wall. The muscle layer with a T2 hypointensity line is suspicious of focal disruption because of the presence of tumors with T2 intermediate signal intensity (thin arrow). In the high $b$ value $(\mathrm{b}=800)$ diffusion-weighted image $(\mathrm{B})$ and apparent diffusion coefficient map (C), the peripheral region of the tumor shows marked diffusion restriction (thick arrow). However, diffusion restriction is not seen at the base of the tumor, including the stalk (asterisk). Additionally, disruption of the muscle layer with intermediate signal intensity is not observed even in high $b$ value images. An axial three-dimensional T1-weighted spoiled gradient echo image (D) obtained 300 seconds after contrast agent administration shows bright delayed enhancement of the tumor stalk (asterisk). This case was preoperatively classified as vesicle imagingreporting and data system grade 2. The patient underwent transurethral resection of the bladder tumor. Appropriate tumor specimens with muscle layer were obtained and it was diagnosed as high-grade stage T1 cancer with invasion only up to the subepithelial connective tissue.

Although a few studies have validated the VI-RADS, this suggests that it is not easy to determine the invasion depth of tumors assigned VI-RADS 3.

One weak point of the VI-RADS is that it does not include significant secondary signs, such as hydroureter caused by ureterovesical junction (UVJ) obstruction, in its scoring. When a $3-\mathrm{cm}$ bladder tumor scored as VI-RADS 3 is located in the dome or near the UVJ causing hydroureter, the treatment approach should be different. A schematic map can also be used to describe the bladder tumor location. The presence of multiple lesions and sizes has also been described (7). However, there are no boundaries between the bladder wall and no formal configuration of the bladder, making it difficult to assign to a single location in the image. Muscle invasiveness and complication rates after TURBT differ by tumor location. Bladder neck cancers have a significantly higher frequency of muscle invasion (23), while bladder dome cancer reportedly has a higher bladder perforation risk than tumors in other bladder wall locations (24).

Although the VI-RADS recommends scoring based on a combination of the three MRI sequences, radiologists should consider using only T2-weighted imaging and diffusion-weighted imaging (biparametric MRI similar to that in prostate MRI) sequences for various reasons when evaluating local $\mathrm{T}$ staging. In fact, previous studies reported that using only T2-weighted imaging plus diffusionweighted imaging showed useful and satisfactory diagnostic accuracy for the T stage $(25,26)$. One recent study reported that fusion of high $b$ value DWI and T2WI using noncontrast MRI can accurately diagnose muscle-invasive 

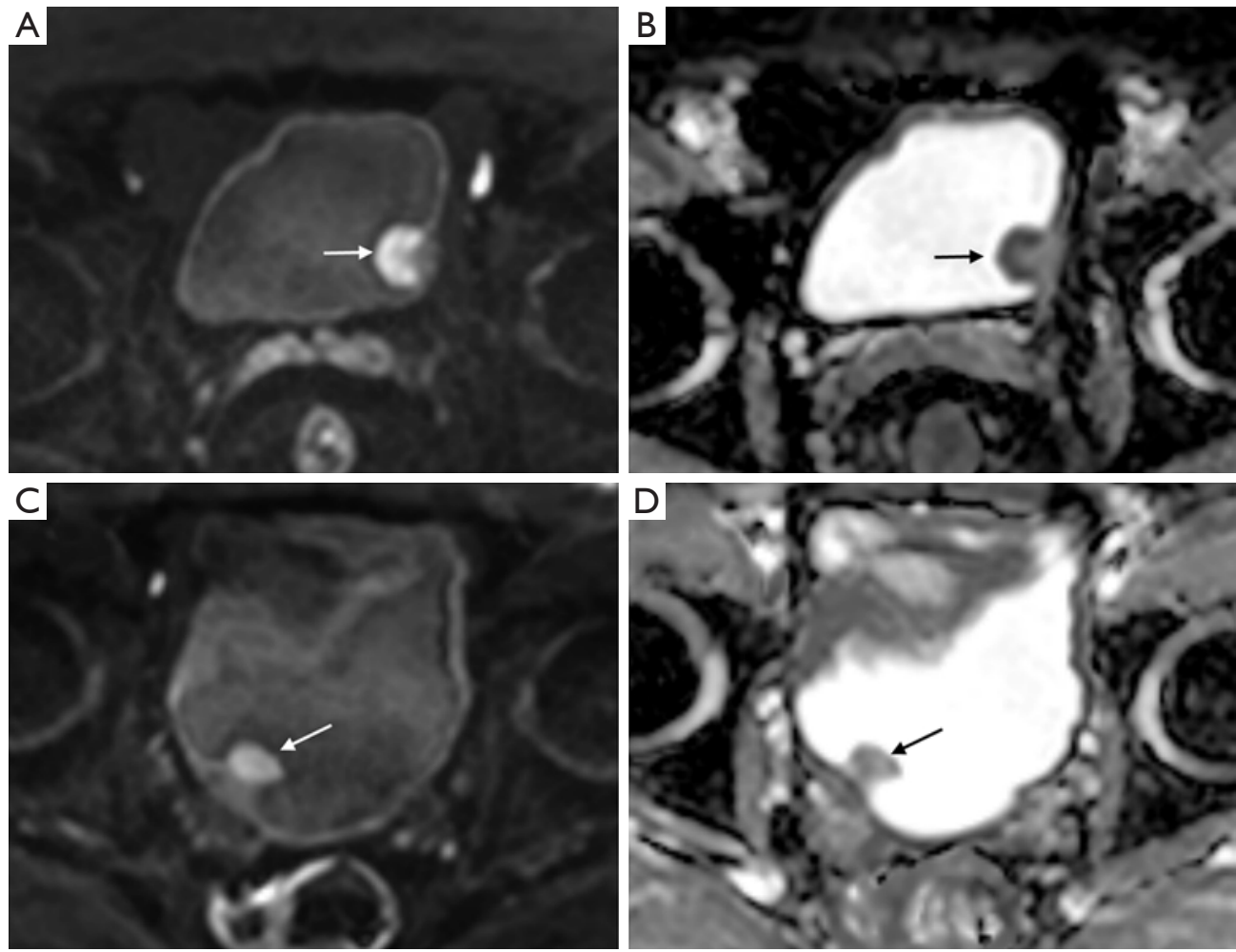

Figure 10 Correlation of apparent diffusion coefficient (ADC) value and tumor grade. (A,B) Urothelial carcinoma (stage T1) in an 82-yearold man. High $b$ value axial diffusion-weighted magnetic resonance image (A) and apparent diffusion coefficient (ADC) map (B) with an ADC value of $0.9 \times 10^{-3} \mathrm{~mm}^{2} / \mathrm{s}$ showing a papillary mass (arrow) in the left wall with marked diffusion restriction. This tumor was staged as a high-grade urothelial carcinoma after the histopathological examination of transurethral resection of bladder tumor (TURBT) specimens. (C,D) Urothelial carcinoma (stage Ta) in a 64-year-old man. High $b$ value axial diffusion-weighted magnetic resonance image (C) and ADC map with an ADC value of $1.45 \times 10^{-3} \mathrm{~mm}^{2} / \mathrm{s}$ (D) showing a papillary mass in right wall with mild to moderate diffusion restriction. The tumor was confirmed as a low-grade urothelial carcinoma on the histopathological examination of TURBT specimens. Both patients underwent diffusion-weighted imaging with the same 3T magnetic resonance imaging equipment (Skyra, Siemens) and parameters; moreover, the window level and width were similar. Although direct comparisons may be inaccurate owing to differences in the cases, a more pronounced diffusion restriction is observed in the diffusion-weighted images of (A) and (B), which were confirmed as high-grade urothelial carcinoma.

tumors (15). According to a recent study that validated VI-RADS, T2-weighted imaging has high sensitivity and diffusion-weighted imaging has high specificity with accuracy (3). In addition, Wang et al. reported little difference in the areas under the receiver operating characteristic curve between mpMRI and biparametric MRI in distinguishing $\mathrm{T} 1$ cancer from T2 cancer (27). Therefore, if biparametric MRI is performed properly, satisfactory $T$ staging is possible without the need for additional imaging $(27,28)$.

Tumor stage determines the local treatment strategy. If the tumor is less than T1 or not bulky, TURBT and intravesical therapy, such as bacille Calmette-Guérin (BCG), or chemotherapy, usually with mitomycin, are commonly used. Upstaging to $\mathrm{T} 2$ at the restage TURBT of an initial T1 tumor occurred in up to $25 \%$ of cases. Moreover, if the initial TURBT specimen does not include a muscle layer, the possibility increased by $45 \%(29,30)$. Thus, the European Association of Urology and American Urological Association guidelines recommend the use of restage TURBT in all $\mathrm{T} 1$ and high-grade tumors. In MIBC and BCG-unresponsive NMIBC, radical cystectomy with pelvic lymph node dissection is considered the standard therapy (31). 

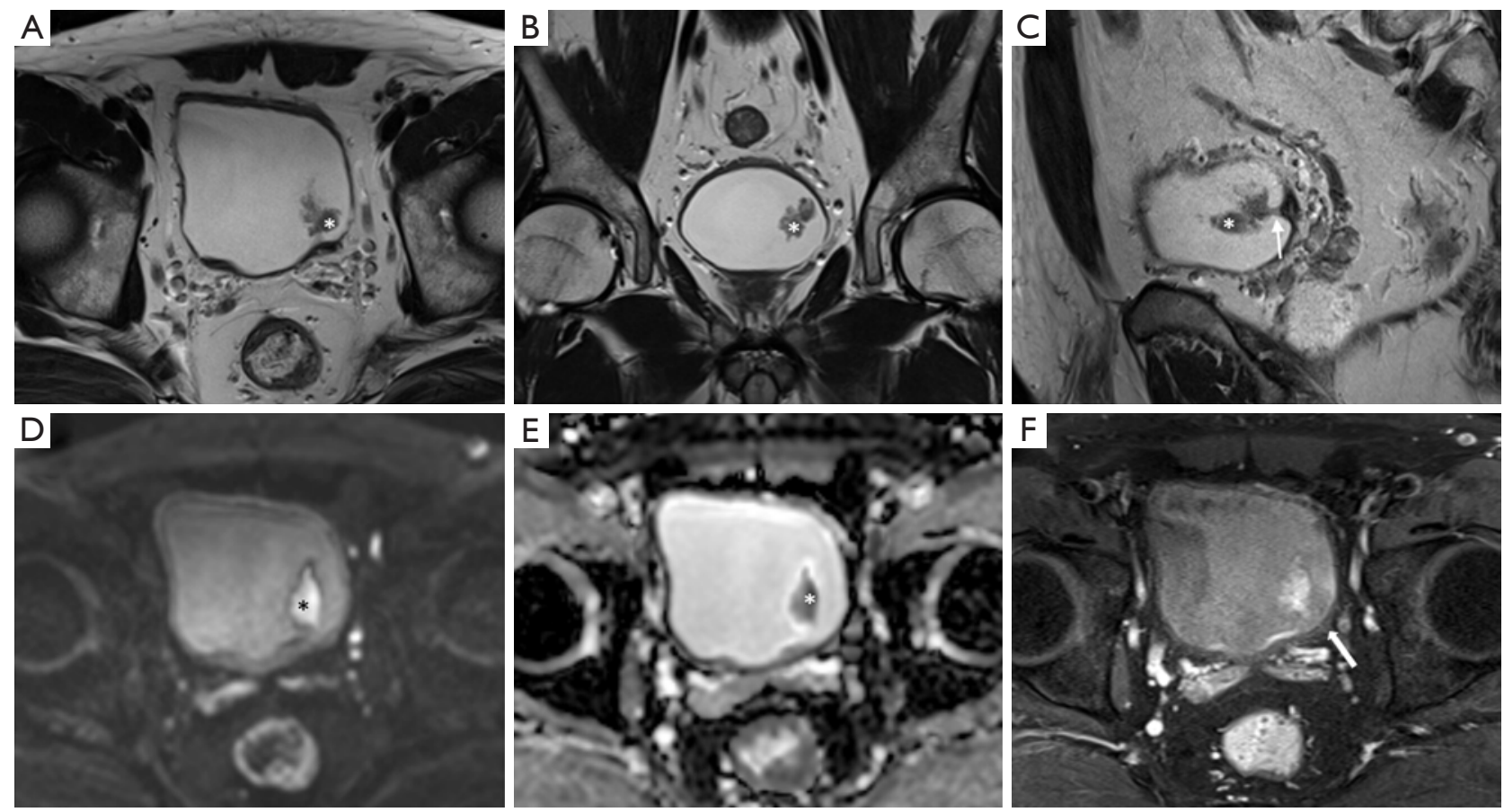

Figure 11 Urothelial carcinoma (stage Ta) in a 56-year-old man. Axial (A), coronal (B), and sagittal (C) T2-weighted images showing an approximately $3-\mathrm{cm}$ papillary mass (asterisk) in the left posterior wall. In axial and coronal images, the tumor appears to float on the bladder. However, the tumor stalk (arrow) is clearly observed in the sagittal image. A high $b$ value diffusion-weighted image (D) and apparent diffusion coefficient map (E) showing the papillary mass with marked diffusion restriction (asterisk). There is no disruption of the muscle layer in the T2WI. Axial three-dimensional T1-weighted spoiled gradient echo image (F) obtained 180 seconds after contrast agent administration showing preserved mild delayed enhancement of the muscle layer (arrow). Therefore, the case can be classified as vesicle imaging-reporting and data system grade 2 .

In the advanced stage, radiation therapy can be used alone or in combination with chemotherapy for palliative purposes. These therapies can also be used for neoadjuvant purposes to shrink the tumor (32).

Usually, $70-80 \%$ of newly diagnosed bladder cancers are NMIBC and the rest are MIBC. NMIBC rarely metastasizes, but recurrence rates of up to $80 \%$ have been reported (16). Depending on grade (high or low) of urothelial carcinoma, progression from NMIBC to MIBC occurs in $10-50 \%$ of cases $(33,34)$; thus, tumor grade is an import predictor of prognosis in NMIBC (4). However, because all muscle-invasive tumors are considered high grade, grading is not considered important for MIBC (12). Instead, differentiating morphological subtypes from pathology can aid with determining prognosis and making treatment decisions (5). MIBC, especially in cases higher than stage $\mathrm{T} 3$, has a poor prognosis because of the presence of occult metastases, including nodal metastases, at the time of diagnosis (16). The lymph nodes in bladder cancer can be divided into primary and secondary drainage sites. The hypogastric, obturator, external iliac, internal iliac, sacral, presacral, and perivesical lymph nodes are the primary drainage sites. These lymph nodes are anatomically confined to the true pelvis and are assigned as $\mathrm{N} 1$ for single node metastasis and $\mathrm{N} 2$ for two or more. The common iliac node is the secondary drainage site; if positive, it is assigned as N3 $(9,22)$. The positive retroperitoneal lymph nodes should be assigned as M1a according to AJCC $8^{\text {th }}$ edition (9).

Previous studies suggested that the possibility of pathologic nodes should be considered in imaging (CT and MRI) when the maximum short-axis diameter is $>8 \mathrm{~mm}$ for pelvic nodes and $>10 \mathrm{~mm}$ for abdominal nodes $(35,36)$. In addition to size, findings such as round morphology, loss of internal architecture, and nodal extracapsular extension suggest more pathologic nodes (7). However, the diagnosis of lymph node metastasis is less accurate and limited on 

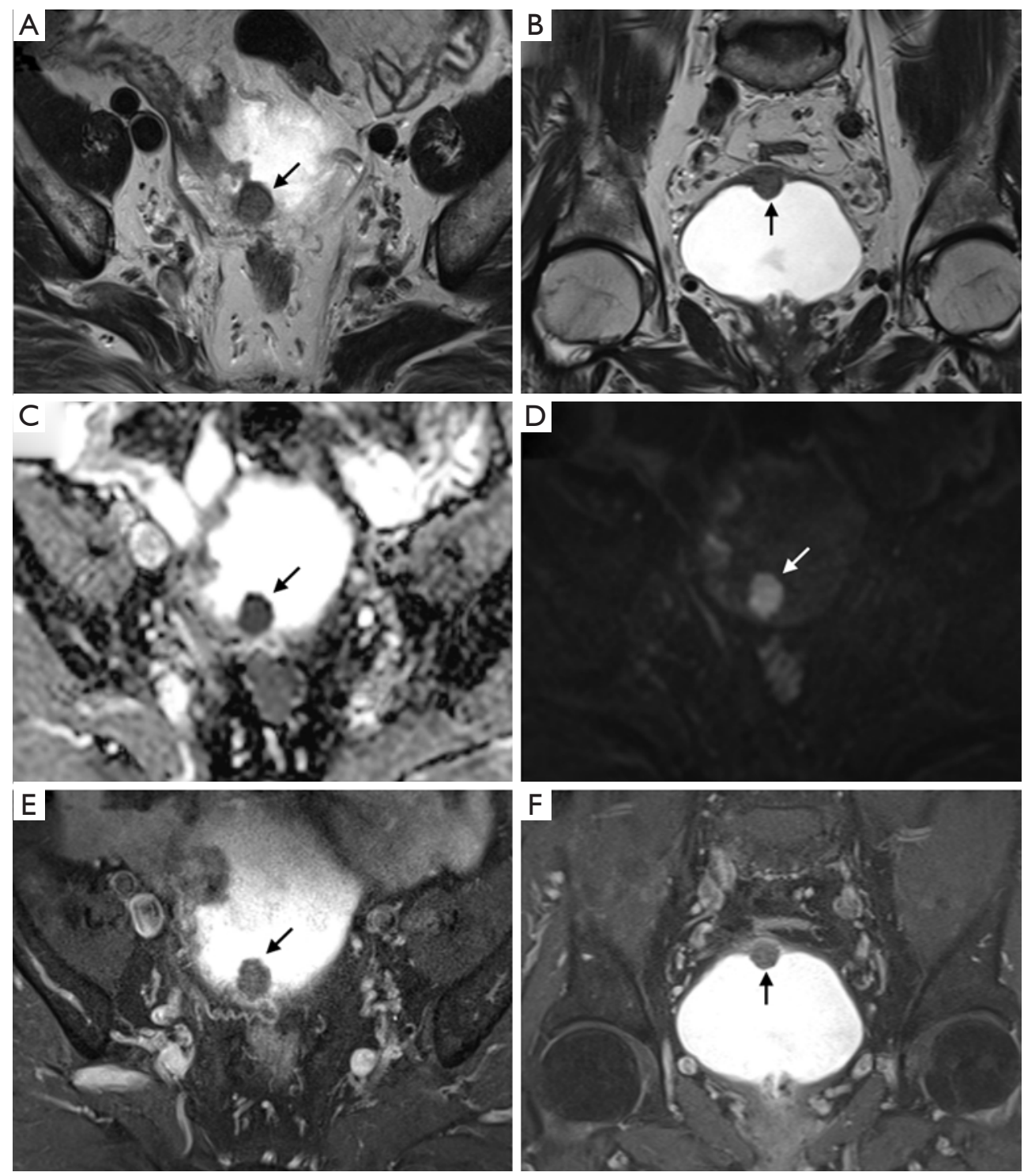

Figure 12 Urothelial carcinoma (stage T2) in an 80-year-old man. Axial (A) and coronal (B) T2-weighted images showing an intermediate signal intensity ovoid-shaped mass (arrow) in the bladder dome. A high $b$ value axial diffusion-weighted magnetic resonance image (C) and apparent diffusion coefficient map (D) showing marked diffusion restriction of the whole tumor area. There is no clear disruption of the low signal intensity muscle layer. However, category 2 findings are lacking on T2-weighted images and diffusion-weighted images. Axial (E) and coronal (F) three-dimensional T1-weighted spoiled gradient echo image obtained 180 seconds after contrast agent administration also show contrast-enhanced category 3 . This case should be scored as vesicle imaging-reporting and data system grade 3 . The patient underwent transurethral resection of the bladder tumor. Appropriate tumor specimens with muscle tissue were examined and the patient was diagnosed with high-grade stage T2 cancer.

imaging modalities because "small lymph nodes with micrometastasis" and "benign lymph node enlargement" are difficult to distinguish. To overcome these drawbacks in cross-sectional imaging, the use of positron emission tomography (PET)-CT and PET-MRI may increase the accuracy of detection of lymph node metastases $(37,38)$. To achieve adequate pathologic nodal staging of bladder cancer, more than 12 lymph node harvests and extended pelvic lymph node dissections, including that of the internal iliac nodes, should be performed (22,39). In recent robotic 

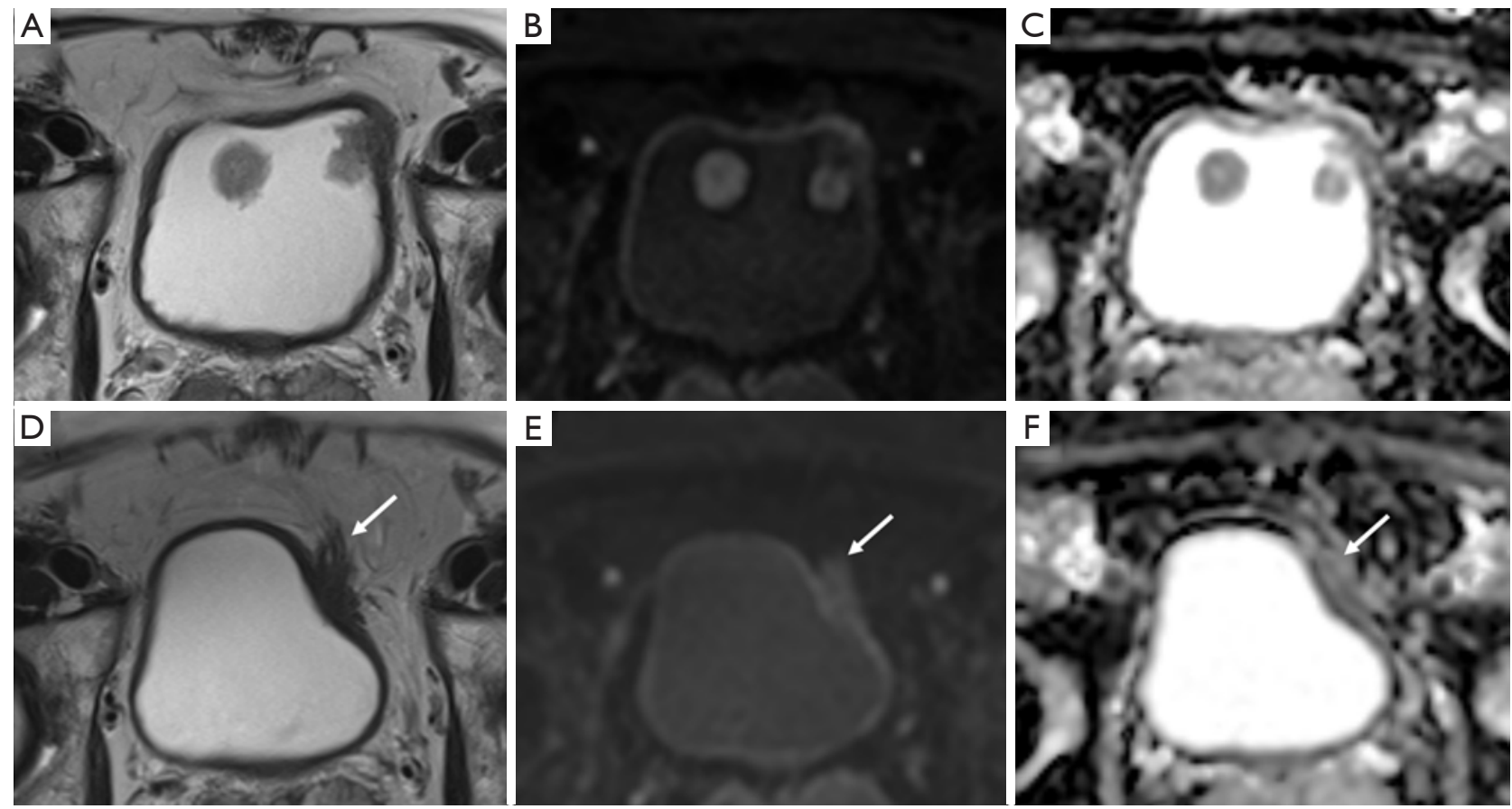

Figure 13 Follow-up magnetic resonance imaging (MRI) of an 82-year-old man who underwent transurethral resection of bladder tumor (TURBT) for urothelial carcinoma. Axial T2-weighted image (T2WI) (A), high $b$ value diffusion-weighted image (DWI) (B), and apparent diffusion coefficient (ADC) map (C) showing two masses in the bladder anterior wall. MRI shows a T2 hypointense bladder cancer without muscle invasion that was diagnosed as high-grade T1 urothelial carcinoma on TURBT specimens including proper muscle tissue. In follow-up MRI, axial T2WI (D), high $b$ value DWI (E), and ADC map (F) obtained 8 months after TURBT showing new perivesical infiltration with hair-like projection (arrow) in the left anterior perivesical area. There was no remarkable diffusion restriction on DWI, and histopathological examination of repeat TURBT specimens containing proper muscle tissue showed acute and chronic inflammation in the adjacent bladder wall.

surgeries in our institution, N3 and M1a node dissections were carefully performed in selective patients. Although not included in the TNM classification, blood and lymphatic vessel invasion are known to have independent prognostic significance (5). The overall 5-year survival rate of localized and superficial bladder tumors exceeds $90 \%$, while that of metastatic bladder cancers is less than $10 \%$; thus, the importance of precise bladder cancer staging cannot be overemphasized (40).

\section{Role of imaging study in post-treatment surveillance}

Although cystoscopy is the gold standard for post-treatment follow-up, noninvasive methods, especially mpMRI, should be used. In addition, simultaneous or alternative followup with CT may be helpful for the identification of upper urinary tract recurrence or distant metastases. However, patients who undergo post-treatment surveillance for bladder cancer often have reduced renal function, thus the repetitive use of MRI or CT contrast agents can be burdensome $(41,42)$. In patients with impaired renal function, biparametric MRI, which does not involve contrast administration, is expected to aid in the diagnosis and staging of local recurrence.

Inflammation after TURBT and fibrosis can cause irregular bladder wall thickening with perivesical infiltration. These changes have similar T2-weighted signal intensity, and it can be challenging to distinguish between perivesical tumor extension and benign conditions $(3,16,43)$. Moreover, post-treatment-related effects can last up to 24 months (44). However, DWI may be useful for identifying tumors on the basis of the presence of restricted diffusion (Figure 13) $(16,45,46)$. DCE sequence is also reportedly useful for post-treatment evaluation (47), but recent comparative studies have shown that diffusion- 
weighted imaging has better diagnostic accuracy, sensitivity, and specificity than DCE (46). The standard treatment for patients with MIBC is radical cystectomy with pelvic lymph node dissection. However, since 1980, neoadjuvant chemotherapy has been used to improve 5 -year survival rates, and mpMRI may play an important role in assessing therapeutic response after neoadjuvant treatment. The evaluation of treatment response in solid tumors is performed by comparing the size change before and after chemotherapy (48); however, since the bladder is not a fixed organ, it is often difficult to apply these criteria. In such cases, if the $\mathrm{ADC}$ value is significantly increased compared with baseline, it can be considered reflective of a response to treatment (49).

\section{Conclusions}

Imaging, particularly MRI, plays a crucial role in the staging of bladder urothelial carcinoma. The use of CT is preferred to evaluate the upper urinary tract, lymph nodes, and distant metastases rather than $\mathrm{T}$ stage. Based on various previous studies, the recently released VI-RADS also focuses on local $\mathrm{T}$ staging using mpMRI. The role of MRI in staging may be important for the diagnosis of small and locally advanced cancers, but it may be more important for differentiating non-muscle invasive disease from muscle-invasive disease. Moreover, the use of various MRI sequences may play an important role in post-treatment surveillance. To ensure a proper evaluation of bladder cancer using MRI, the acquisition of images using an appropriate protocol and adequate patient preparation is necessary. The appropriate use of VI-RADS is also recommended since it is thought to aid communication among radiologists, urologists, and other medical staff.

\section{Acknowledgments}

Funding: None.

\section{Footnote}

Provenance and Peer Review: This article was commissioned by the Guest Editors (Ja Hyeon Ku, Ho Kyung Seo, Seok Ho Kang) for the series "Muscle-Invasive Bladder Cancer" published in Translational Andrology and Urology. The article has undergone external peer review.

Conflicts of Interest: Both authors have completed the ICMJE uniform disclosure form (available at http://dx.doi. org/10.21037/tau-19-671). The series "Muscle-Invasive Bladder Cancer" was commissioned by the editorial office without any funding or sponsorship. The authors have no other conflicts of interest to declare.

Ethical Statement: The authors are accountable for all aspects of the work in ensuring that questions related to the accuracy or integrity of any part of the work are appropriately investigated and resolved.

Open Access Statement: This is an Open Access article distributed in accordance with the Creative Commons Attribution-NonCommercial-NoDerivs 4.0 International License (CC BY-NC-ND 4.0), which permits the noncommercial replication and distribution of the article with the strict proviso that no changes or edits are made and the original work is properly cited (including links to both the formal publication through the relevant DOI and the license). See: https://creativecommons.org/licenses/by-nc-nd/4.0/.

\section{References}

1. Bray F, Ferlay J, Soerjomataram I, et al. Global cancer statistics 2018: GLOBOCAN estimates of incidence and mortality worldwide for 36 cancers in 185 countries. CA Cancer J Clin 2018;68:394-424.

2. Wong-You-Cheong JJ, Woodward PJ, Manning MA, et al. Neoplasms of the urinary bladder: radiologic-pathologic correlation. Radiographics 2006;26:553-80.

3. Wang H, Luo C, Zhang F, et al. Multiparametric MRI for bladder cancer: validation of VI-RADS for the detection of detrusor muscle invasion. Radiology 2019;291:668-74.

4. Soukup V, Čapoun O, Cohen D, et al. Prognostic performance and reproducibility of the 1973 and 2004/2016 World Health Organization Grading classification systems in non-muscle-invasive bladder cancer: a European Association of Urology non-muscle invasive bladder cancer guidelines panel systematic review. Eur Urol 2017;72:801-13.

5. Witjes J, Bruins M, Compérat E, et al. EAU guidelines muscle invasive bladder cancer 2018. EAU Annual Congress Copenhagen, 2018.

6. Verma S, Rajesh A, Prasad SR, et al. Urinary bladder cancer: role of MR imaging. Radiographics 2012;32:371-87.

7. Panebianco V, Narumi Y, Altun E, et al. Multiparametric magnetic resonance imaging for bladder cancer: development of VI-RADS (Vesical Imaging-Reporting And Data System). Eur Urol 2018;74:294-306. 
8. Kim SH. Radiology Illustrated: Uroradiology. Berlin, Heidelberg: Springer Science \& Business Media, 2011.

9. Paner GP, Stadler WM, Hansel DE, et al. Updates in the eighth edition of the tumor-node-metastasis staging classification for urologic cancers. Eur Urol 2018;73:560-9.

10. American Urological Association. Urinary Bladder: Lamina Propria. Available online: https://www.auanet. org/education/auauniversity/education-products-andresources/pathology-for-urologists/normal-histology-andimportant-histo-anatomic-structures/urinary-bladderlamina-propria

11. McKibben MJ, Woods MEJCur. Preoperative imaging for staging bladder cancer. Curr Urol Rep 2015;16:22.

12. Witjes JA, Compérat E, Cowan NC, et al. EAU guidelines on muscle-invasive and metastatic bladder cancer: summary of the 2013 guidelines. Eur Urol 2014;65:778-92.

13. Woo S, Suh CH, Kim SY, et al. Diagnostic performance of MRI for prediction of muscle-invasiveness of bladder cancer: a systematic review and meta-analysis. Eur J Radiol 2017;95:46-55.

14. Hayashi N, Tochigi H, Shiraishi T, et al. A new staging criterion for bladder carcinoma using gadoliniumenhanced magnetic resonance imaging with an endorectal surface coil: a comparison with ultrasonography. BJU Int 2000;85:32-6.

15. Lee M, Shin SJ, Oh YT, et al. Non-contrast magnetic resonance imaging for bladder cancer: fused high $\mathrm{b}$ value diffusion-weighted imaging and T2-weighted imaging helps evaluate depth of invasion. Eur Radiol 2017;27:3752-58.

16. Lee CH, Tan CH, Faria SC, et al. Role of Imaging in the Local Staging of Urothelial Carcinoma of the Bladder. AJR Am J Roentgenol 2017;208:1193-205.

17. Yu RJ, Stein JP, Cai J, et al. Superficial (pT2a) and deep (pT2b) muscle invasion in pathological staging of bladder cancer following radical cystectomy. J Urol 2006;176:493-8; discussion 498-9.

18. Ghafoori M, Shakiba M, Ghiasi A, et al. Value of MRI in local staging of bladder cancer. Urol J 2013;10:866-72.

19. Rabie E, Faeghi F, Izadpanahi MH, et al. Role of dynamic contrast-enhanced magnetic resonance imaging in staging of bladder cancer. J Clin Diagn Res 2016;10:TC01-5.

20. Narumi Y, Kadota T, Inoue E, et al. Bladder wall morphology: in vitro MR imaging-histopathologic correlation. Radiology 1993;187:151-5.

21. Al Johi RS, Seifeldein GS, Moeen AM, et al. Diffusion weighted magnetic resonance imaging in bladder cancer, is it time to replace biopsy? Cent European J Urol 2018;71:31.

22. Edge S, Byrd D, Compton C, et al. AJCC Cancer Staging Manual 7th Edition. Chicago: Springer, 2010.

23. Xiao GQ, Rashid HJ. Bladder neck urothelial carcinoma: a urinary bladder subsite carcinoma with distinct clinicopathology. Int J Surg Pathol 2015;23:517-23.

24. Bansal A, Sankhwar S, Goel A, et al. Grading of complications of transurethral resection of bladder tumor using Clavien-Dindo classification system. Indian J Urol 2016;32:232.

25. Takeuchi M, Sasaki S, Ito M, et al. Urinary bladder cancer: diffusion-weighted MR imaging accuracy for diagnosing T stage and estimating histologic grade. Radiology 2009;251:112-21.

26. Wu LM, Chen XX, Xu JR, et al. Clinical value of T2weighted imaging combined with diffusion-weighted imaging in preoperative $T$ staging of urinary bladder cancer: a large-scale, multi-observer prospective study on 3.0-T MRI. Acad Radiol 2013;20:939-46.

27. Wang HJ, Pui MH, Guo Y, et al. Multiparametric 3-T MRI for differentiating low-versus high-grade and category T1 versus T2 bladder urothelial carcinoma. AJR Am J Roentgenol 2015;204:330-4.

28. Sakamoto K, Ito M, Ikuta S, et al. Detection of muscleinvasive bladder cancer on biparametric MRI using Vesical Imaging-Reporting and Data System and apparent diffusion coefficient values (VI-RADS/ADC). Bladder Cancer 2020;6:161-9.

29. Manoharan V, Mavuduru RS, Kumar S, et al. Utility of restage transurethral resection of bladder tumor. Indian J Urol 2018;34:273.

30. Herr HW, Donat SM. Quality control in transurethral resection of bladder tumours. BJU Int 2008;102:1242-6.

31. Davis JW, Gaston K, Anderson R, et al. Robot assisted extended pelvic lymphadenectomy at radical cystectomy: lymph node yield compared with second look open dissection. J Urol 2011;185:79-83.

32. James ND, Hussain SA, Hall E, et al. Radiotherapy with or without chemotherapy in muscle-invasive bladder cancer. New Eng J Med 2012;366:1477-88.

33. Millán-Rodríguez F, Chéchile-Toniolo G, Salvador-Bayarri $\mathrm{J}$, et al. Multivariate analysis of the prognostic factors of primary superficial bladder cancer. J Urol 2000;163:73-8.

34. Nepple KG, O’Donnell MA. The optimal management of T1 high-grade bladder cancer. Can Urol Assoc J 2009;3:S188.

35. Barentsz JO, Engelbrecht MR, Witjes JA, et al. MR 
imaging of the male pelvis. Eur Radiol 1999;9:1722-36.

36. Dorfman RE, Alpern MB, Gross B, et al. Upper abdominal lymph nodes:criteria for normal size determined with CT. Radiology 1991;180:319-22.

37. Rosenkrantz AB, Friedman KP, Ponzo F, et al. Prospective pilot study to evaluate the incremental value of PET information in patients with bladder cancer undergoing 18F-FDG simultaneous PET/MRI. Clin Nucl Med 2017;42:e8.

38. Margolis DJ, Hu JC. Vying for standardization of bladder cancer MRI interpretation and reporting: VI-RADS. Radiology 2019;291:675-6.

39. Campi R, Sessa F, Di Maida F, et al. Templates of lymph node dissection for renal cell carcinoma: a systematic review of the literature. Front Surg 2018;5:76.

40. Gschwend JE, Dahm P, Fair WR. Disease specific survival as endpoint of outcome for bladder cancer patients following radical cystectomy. Eur Urol 2002;41:440-8.

41. Beam AS, Moore KG, Gillis SN, et al. GBCAs and risk for nephrogenic systemic fibrosis: a literature review. Radiol Technol 2017;88:583-9.

42. Mohammed NMA, Mahfouz A, Achkar K, et al. Contrastinduced nephropathy. Heart Views 2013;14:106-16.

43. Yoshida S, Koga F, Kawakami S, et al. Initial experience of diffusion-weighted magnetic resonance imaging to assess therapeutic response to induction chemoradiotherapy

Cite this article as: Sim KC, Sung DJ. Role of magnetic resonance imaging in tumor staging and follow-up for bladder cancer. Transl Androl Urol 2020;9(6):2890-2907. doi: 10.21037/ tau-19-671 against muscle-invasive bladder cancer. Urology 2010;75:387-91.

44. Johnson RJ, Carrington BM, Jenkins J, et al. Accuracy in staging carcinoma of the bladder by magnetic resonance imaging. Clin Radiol 1990;41:258-63.

45. Takeuchi M, Sasaki S, Naiki T, et al. MR imaging of urinary bladder cancer for T-staging: a review and a pictorial essay of diffusion-weighted imaging. J Magn Reson Imaging 2013;38:1299-309.

46. Wang HJ, Pui MH, Guo Y, et al. Diffusion-weighted MRI in bladder carcinoma: the differentiation between tumor recurrence and benign changes after resection. Abdom Imaging 2014;39:135-41.

47. Donaldson SB, Bonington SC, Kershaw LE, et al. Dynamic contrast-enhanced MRI in patients with muscle-invasive transitional cell carcinoma of the bladder can distinguish between residual tumour and postchemotherapy effect. Eur J Radiol 2013;82:2161-8.

48. Eisenhauer EA, Therasse P, Bogaerts J, et al. New response evaluation criteria in solid tumours: revised RECIST guideline (version 1.1). Eur J Cancer 2009;45:228-47.

49. Hafeez S, Koh M, Sohaib A, et al. PD-0464: assessing response to chemotherapy with diffusion weighted MRI (DW-MRI) in muscle invasive bladder cancer (MIBC). Radiother Oncol 2013;106:S180-1. 